\title{
Positron Emission Tomography (PET) in Clinical Medicine and Bangladesh Perspective
}

\author{
MSH MAJUMDER
}

\begin{abstract}
Summary:
Positron emission tomography (PET) imaging devices were first developed in the 1970. In the last thirty years PET imaging has been extensively used in research, but only in the last few years the technology has become more widely available for clinical applications. Most of the early works with PET focused on brain metabolism, partly because of the smaller size of detector needed to study head. With the introduction of improved instruments and suitable radionuclide, applications in oncology have developed into the major clinical uses of PET. Myocardial viability is usually assessed using perfusion studies with Signal photon emission computed tomography (SPECT) but in case of inconclusive result, metabolic assessment with short-lived PET tracers may be more decisive. PET allows study of body function; it helps detection of alterations in biochemical processes that suggest disease before changes in anatomy are apparent with other imaging tests, such as computed tomography (CT) or magnetic resonance imaging (MRI).
\end{abstract}

Introduction:

The existence of the positron in the atom was predicted by British physicist Paul Dirac in the year 1928. Rutherford (1910) established solar atomic model - a positively charged nucleus surrounded by a cloud of negatively charged electrons; later, it was redefined by Bohr (1913) that stated electrons travel in discrete orbits circling around the dense nucleus. Paul Dirac arrived at his prediction by applying the theory of relativity of physicist Albert Einstein to observations of the motion of particles with electric charge. Based on Einstein's theory and mathematical analyses of the motion of electrons, Dirac assumed the existence of a new kind of particle that was identical to the electron in all ways except the sign of its electric charge $e^{1,2}$. Dirac's theoretical particle received the name positron, although

Address of Correspondence: Md. Shafayet Hasan Majumder, MBBS, M.Phil, FCPS, Associate Professor, Department of Medicine, Medical College for Women and Hospital, Uttara Model Town, Uttara, Dhaka-1213, Mobile: 01720172992, Tel: 9665257, E-mail: shafayet57@hotmail.com

Received: 8 August 2010

Accepted: 20 June 2011
The future of PET imaging is bright. New geometries are being studied especially to develop organ specific imaging devices, new detector materials are being developed and techniques for reconstruction are improving. However, perhaps the most important need for further utilization of PET imaging is the development of new radiopharmaceuticals or radiotracer compounds and better understanding of cellular physiology and metabolism in disease states. Until recently, Bangladesh had no positron emission tomography scanner, though the neighboring countries like India, Pakistan, Thailand \& Singapore have PET scanning for last several years. PET scanning in Bangladesh has been introduced by a multidisciplinary tertiary level private hospital - United Hospital, Gulshan, Dhaka recently.

Key Words: Position emission tomography Positron 18 Flurodeoxyglucose

(J Bangladesh Coll Phys Surg 2011; 29: 151-157)

the scientific community was reluctant to accept this finding. This reluctance was vanished in 1932, when American physicist Carl David Anderson discovered the same particle while tracking the paths of subatomic particles. Some of the paths Anderson analyzed had the same curve as electron paths but with positive charge. Thus, he indicated the existence of particles that possessed the characteristics Dirac had predicted earlier mathematically ${ }^{2,3}$.

Positron is an elementary or fundamental particle identical to the electron except for its electric charge and its magnetic moment (a property that determines how it behaves in a magnetic field); and it can not be divided into smaller units. All elementary particles have basic characteristics called mass, charge and spin. The positron has the same mass - amount of matter as the electron, and the same spin. The two particles also have the same amount of electric charge, but the positron's charge is positive and the electron's is negative. For this reason, the positron is sometimes called a positive electron. Although positrons and electrons have a measurable mass, charge, and spin, 
they have no measurable size, shape, or structure. They are considered as point like. Other point like elementary particles includes neutrinos and quarks. Every elementary particle has an equal and opposite antiparticle. The positron is the antiparticle of the electron. Just as particles combine to form ordinary matter, antiparticles combine to create antimatter. When a particle and its antiparticle collide, they destroy each other releasing energy. This feature makes positron useful in creating Positron Emission Scan. Tomography is a special X-ray or imaging technique that blurs out the shadows of superimposed structures to show more clearly the principal structures being examined. To create a Positron Emission Tomography scan, positron emitting substances are injected into the body. Computers track the energy released inside the body by positron- electron collisions and use this information to form images. Positrons, as positron emission tomography scan, have uses in medicine and in industry. PET scans, as shown in Figure-1A \& B are especially helpful in identifying and locating brain tumours, lesions in brain and other parts of the body ${ }^{2}$, $3,4,5,6$.

Positron sources can be natural as well as artificial. Positrons are naturally produced within stars and by collision of cosmic rays. They can also be produced artificially, called positron-emitting radionuclide in the laboratory in a cyclotron machine by interaction of gamma rays with a very heavy atom ${ }^{2}$. The radionuclide emit positron which after moving a very short distance,

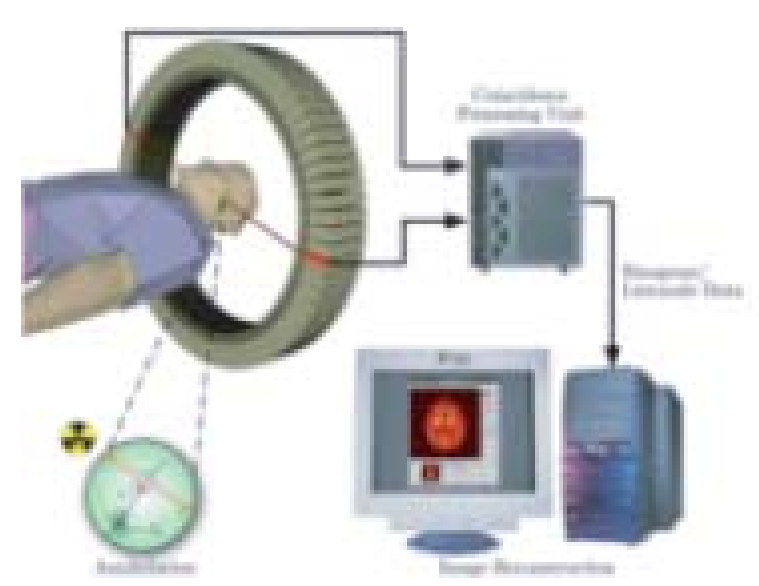

Fig.-1A: PET acquisition process.

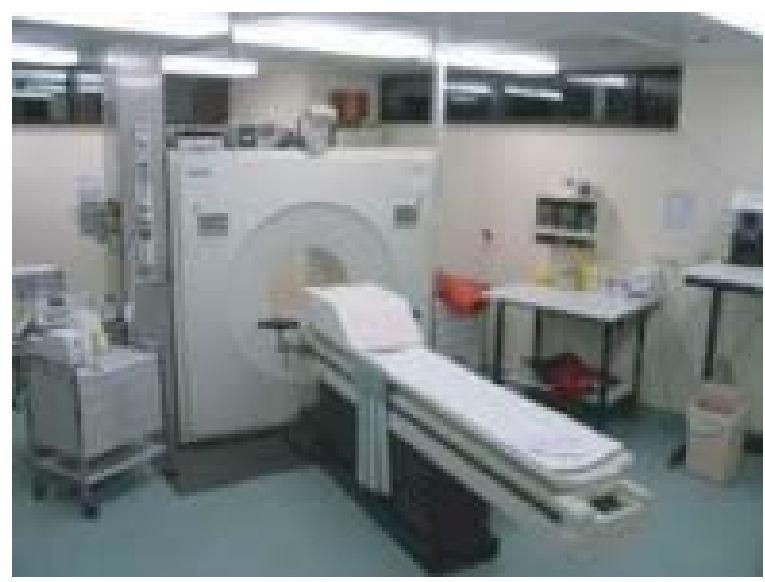

Fig.-B: Typical PET facility.

encounter and combine a nearby electron; and destroy each other. The destruction of both positron and electron leads to simultaneous production of two $511 \mathrm{kev}$ (killoelectron volt) photons that travel in opposite direction at an angle of $180^{\circ}$. This is called annihilation reaction or radiation( Figure 2). Such coincident pairs of photons are to be recognized by detector placed on opposite sides of position of source of radiation ${ }^{2,3}$.

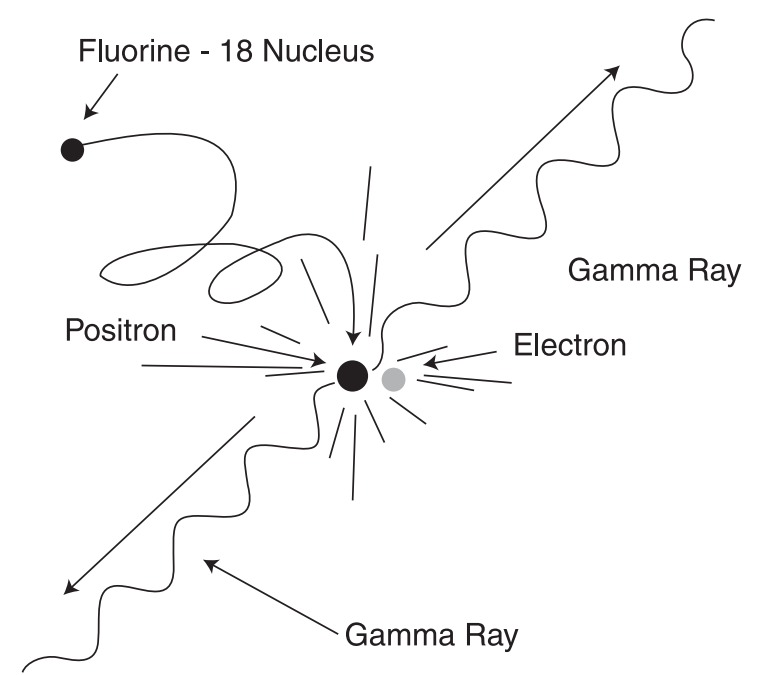

Fig.-2: Positron emission and annihilation radiation, producing 2 gamma photons which travel in opposite direction.

Radiopharmaceutical Development For 'PET’ Imaging Positron emitting radionuclides were discovered in the 1940. The only isotopes of carbon, nitrogen, and oxygen that are suitable for imaging in vivo are the positron 
emitters such as ${ }^{11} \mathrm{C},{ }^{13} \mathrm{~N}$, and ${ }^{15} \mathrm{O}$. Unfortunately, all these nuclides are short lived, so that they can only be used in close proximity to the cyclotron in which they are produced. Even so, they have been used to label a wide range of naturally occurring small organic molecules for physiological research and larger molecules for pharmacological investigations, but none are yet in routine clinical use. PET imaging was initially based on the use of ${ }^{15} \mathrm{O}$ labeled to $\mathrm{O}_{2}$, $\mathrm{CO}$ and $\mathrm{CO}_{2}$ primarily because the Allis Chalmers cyclotron was a deuteron machine and was primarily used to producing ${ }^{15} \mathrm{O} .{ }^{15} \mathrm{O}$ labeled carbon monoxide (CO) provided a means of measuring regional blood volume and blood flow in research tool. Ter-Pogossian and Power had demonstrated that ${ }^{15} \mathrm{O}$ labeled water could be used to measure blood flow in brain and other organs long before PET was developed ${ }^{7,8}$.

One of the prime reasons for the importance of PET in medical research and clinical practice is the existence of positron emitting isotopes of elements such as carbon, nitrogen, oxygen and fluorine which may be processed to create a range of tracer compounds similar to naturally occurring substances in the body. The tracer compounds are carbon monoxide, carbon dioxide, water, methionine, ammonia, flurodeoxyglucose, etc. The physiological processes or functions involved by these radiotracer compounds are blood perfusion, protein synthesis, glucose metabolism, receptor binding's etc ${ }^{9}$. One of the factors most responsible for the acceptance of positron imaging was the development of radiopharmaceuticals, particularly the development of ${ }^{18} \mathrm{~F}$ labeled flurodeoxyglucose (18-FDG), which expanded the scope of PET imaging (Figure 3). Flurodeoxyglucose is basically a glucose analogue molecule, where one oxygen atom is replaced by an atom of fluorine ${ }^{9,10}$. Currently, the mainstay of clinical PET is deoxyglucose labeled with Fluorine-18 (18-FDG). In a normal subject about half of the injected dose is excreted in the urine in 2 to 2.5 hours after injection. The half-life of Fluorine-18 is 110 minutes, so a daily production is required, and the location of imaging needs to be within 1 to 2 hours of the cyclotron production site. Absolute measurement of glucose uptake into lesions or specific organs is difficult because of differences in the size and body composition of the patients, and individual variations in glucose metabolism. Approximate quantitation is achieved by using the Standardized Uptake Value (SUV), which is an estimate of the uptake of FDG into the lesion or organ of interest compared with the mean uptake in the rest of the body ${ }^{8}$.

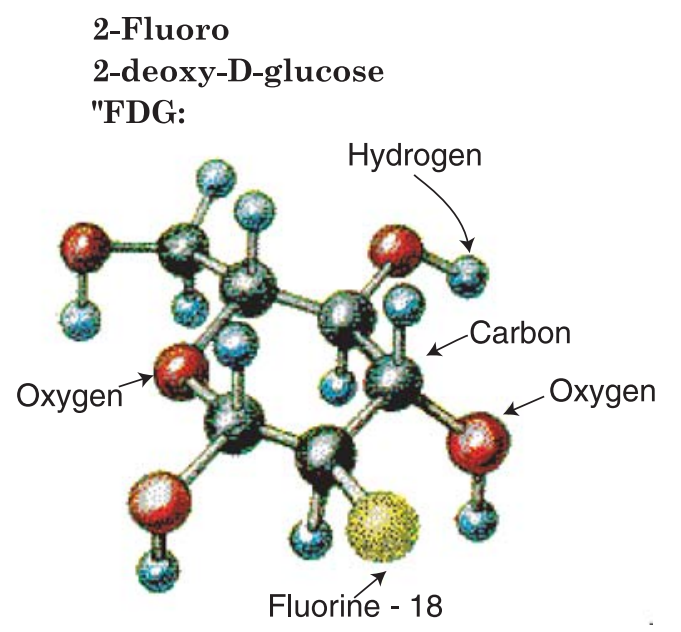

Fig.-3. 18-FDG, an analogue of glucose molecule, where an oxygen atom is replaced by a fluorine atom which is radioactive.

\section{Instrumental Development for Pet Imaging}

The first application of positron annihilation radiation for medical imaging is well documented in the early 1950s by a simple positron scanner using two opposed sodium iodide detectors (Figure-4).

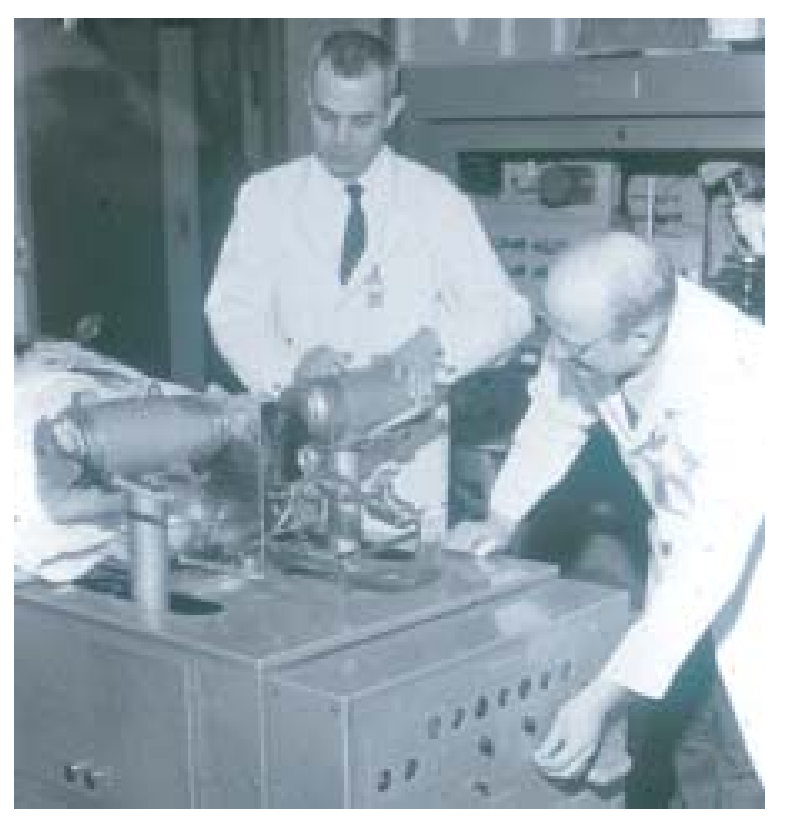

Fig.-4: First clinical positron imaging device 
Several versions of the single pair of coincidence system were built and a hybrid scanner was developed in mid 1960s and the scanner was designed specifically for brain imaging and served that purpose for nearly a decade. Latter on, positron camera (PC-I), PC-II, PCR-I single ring and PCRII cylinder have been developed (Figure-5).

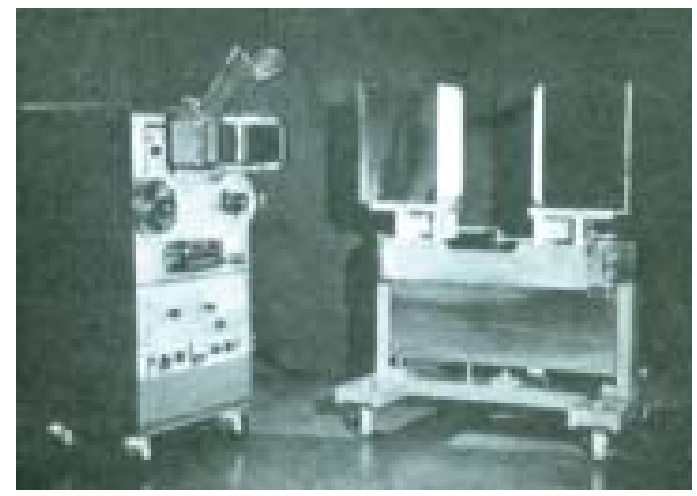

Fig.-5: Hybrid positron scanner.

The recent introduction of luteum-based detectors, together with faster electronics in PET offers a further increase in sensitivity, so that examination times for whole body examination can be considerably reduced ${ }^{11,} 12,13$. Using a dual or multiheaded gamma camera for PET has the obvious advantages that the same instrument can also be used for single photon imaging as well, but the performance of these systems is inferior to dedicated PET instruments. One of the major limitations of PET is lack of anatomical landmarks, particularly in the thorax, abdomen and pelvis and therefore PET images need to be interpreted in conjunction with CT or MRI anatomy for application in oncology ${ }^{8}$.

PET-CT is the fusion of functional and anatomical information acquired almost simultaneously that lets us see the body and disease in a way that is diagnostically very useful (Figure-6).
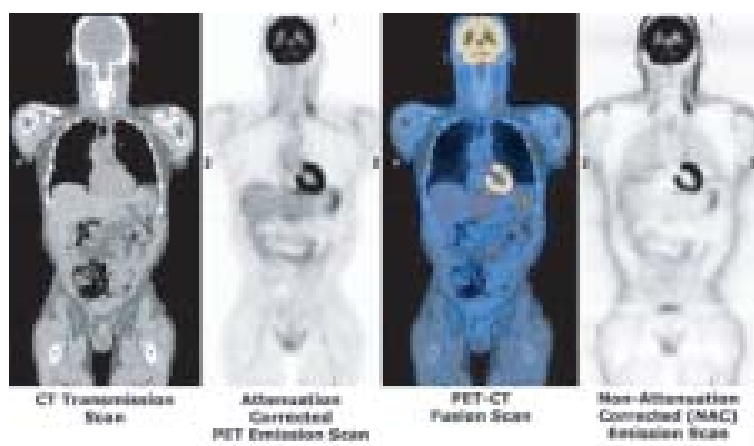

Fig.-6: The CT scan, attenuation corrected PET emission scan, PET-CT fusion scan and Nonattenuation corrected (NAC) Emission scan.
By combining the structural anatomic information with functional data, we are able to visualize form and function. An understanding of the normal and benign as well as the pitfalls and artifacts is essential for accurate information. The PET-CT scanner is essentially full ring coincidence detectors, the PET portion, physically mounted together with CT system of various types. Usually the CT transmission scan is acquired followed by the emission PET scan. Because the scanners are in the same gantry the patient remains on a single scanning table and in the same position for both parts of the scan. Therefore, they are intrinsically registered as seen on the fusion image. Images are presented and viewed in 3 planes (coronal, transaxial and sagittal) as well as a 3D maximum intensity projection, which can be rotated while viewing. The CT, corrected and uncorrected PET scans, as well as the fusion image can be viewed on a single page. Typically, the CT acquisition is performed first, followed by PET acquisition. The anatomical CT data can then be used to apply attenuation correction for the PET data and the resulting images can be displayed separately or fused together ${ }^{\mathbf{4}, \mathbf{8}}$.

\section{PET Applications}

Much of the early works with PET focused on brain metabolism, partly because of the smaller size of detector needed to study head. With the introduction of improved instruments allowing acquisition of whole body images in under an hour, applications in oncology, together with other areas, have developed into the major clinical use of PET.

Oncology: Over 90\% of all PET scans in current practice are done in the field of oncology. Cancerous tissue has deranged glucose metabolism. Glucose uptake and glycolysis proceed about ten times faster in most solid tumors than in non-cancerous tissue. Tumor cells commonly experience hypoxia because they initially lack an extensive capillary network to supply the tumour with oxygen. PET scanning with radiotracer 18 FDG is widely used in clinical oncology. This radiotracer is a glucose analogue that is taken up by glucose-using cell and phosphorylated by hexokinase. Because the oxygen atom replaced by 18-F to generate FDG is required for the next step in glucose metabolism in all cells, no further reactions occur. This means that FDG is trapped in any cell which takes it up, since phosphorylated sugars cannot exit from the cell. This results in intense labeling of tissues with high glucose uptake, such as the brain, 
the liver, and most caners. As a result FDG-PET can be used for diagnosis, staging, and monitoring treatment of cancer, particularly in Hodgkin's disease, nonHodgkin's lymphoma, lung cancer, head and neck cancer.

Most types of lymphoma show avid uptake of FDG, and is suggested that those patient with most intense uptake at presentation have the worst prognosis. When PET has been used as a staging procedure for lymphoma, it has proved to be more accurate than CT. PET is more likely to determine the presence or absence of disease in lymph nodes that are close to the normal size limits. A fall in FDG uptake which may be seen after 1-2 cycles of chemotherapy is a useful predictor of clinical response. In patient with residual mass after radiotherapy and/or chemotherapy, PET is a fairly good predictor of future recurrence. Small cell lung cancer is usually multifocal at the time of presentation, so surgery is rarely indicated. Since systemic chemotherapy is the mainstay of treatment, precise staging is less important than in surgical cases, and the use of FDG-PET is of limited value. Non-small cell lung cancer is much more likely to be amenable to surgery, so a careful staging is needed. FDG-PET is highly accurate in staging mediastinal lymph nodes in this condition, and is at least as accurate as the combination of CT scan and bone scintigraphy for detecting metastasis in the abdomen, pelvis and skeleton. Residual soft tissue abnormality after treatment may be non-specific on CT, and in this condition a negative FDG-PET study may eliminate the need for biopsy. About one half of non-calcified, solitary pulmonary nodules which present for investigations turn out to be benign. FDG-PET offers a non-invasive method for discriminating between benign and malignant nature with sensitivity about 95\% and specificity greater than $80 \%$, so a negative PET result is more reassuring than a negative biopsy. However, because individual scans are more expensive than conventional imaging with CT and MRI, expansion of FDG-PET in cost constrained health services will depend on proper health technology assessment ${ }^{5}$, $9,15,16,17$.

Neurology: PET neuroimaging is based on an assumption that areas of high radioactivity are associated with brain activity. What is actually measured indirectly is the flow of blood to different parts of the brain, which is generally believed to be correlated, and has been measured using the tracer oxygen $\left({ }^{15} \mathrm{O}\right)$. However, because of its 2 -minute half-life, ${ }^{15} \mathrm{O}$ must be piped directly from medical cyclotron for such uses, and this is difficult. In practice, the brain is normally a rapid user of glucose, and since brain pathology, such as in Alzheimer's Disease (AD), greatly decreases brain metabolism of both glucose and oxygen, standard FDGPET of the brain which measures regional glucose use may also be successfully used to differentiate Alzheimer's disease from other dementing processes, and also to make early diagnosis of Alzheimer's disease. Several radiotracers have been developed for PET that are ligands for specific neruroreceptor subtypes (e.g. dopamine D2, serotonin 5-HT1 A, etc) or enzyme substrates (e.g. 6-FDOPA for the AADC enzyme). These agents permit the visualization of neuroreceptor pools in the context of a plurality of neuropsychiatry and neurologic illnesses. A novel probe developed at the University of Pittsburgh, USA termed Pittsburgh compound-B (PIB) permits the visualization of amyloid plaques in the brains of Alzheimer's patients. This technology could assist clinicians in making a positive clinical diagnosis of AD pre-mortem and aid in the development of novel anti-amyloidal therapies ${ }^{8,14}$.

Cardiology: PET imaging utilizing FDG and other perfusion tracers provides valuable diagnostic and prognostic information in patients with ischemic left ventricular dysfunction and has comparable accuracy to competing technologies for detection of viability. In clinical cardiology, FDG-PET can identify so called hibernating myocardium but its cost effectiveness in this role versus SPECT is unclear $4,17,18$.

Neuropsychology and Psychiatry: This aspect is to examine links between specific psychological processes or disorders and brain activity. Numerous compounds that bind selectively to neruroreceptors of interest in biological psychiatry have been radio labeled with ${ }^{11} \mathrm{C}$ or ${ }^{18} \mathrm{~F}$. Radioligands that bind to dopamine receptors (mu) and other sites have been used successfully in studies with human subject including disease such as schizophrenia, substance abuse, mood disorders and other psychiatric conditions ${ }^{4}$.

Pharmacology: In pre-clinical trials it is possible to radio label a new drug and inject it into animals. The uptake of the drug, the tissues in which it concentrates and its eventual elimination, can be monitored far more quickly and cost effectively than the older technique of killing 
and dissecting the animals to discover the same information. PET scanners for rats and apes are marketed for this purpose. Drug occupancy at the purported site of action can also be inferred indirectly by competition studies between unlabeled drug and radio labeled compounds known apriori to bind with specificity to the site ${ }^{4}$.

\section{PET Scans Safety:}

PET scanning is non-invasive, but it does involve exposure to ionizing radiation. The total dose of radiation is small, however, usually around $7 \mathrm{mSv}$ (milisevdvergh, radiation dose unit). This can be compared to $2.2 \mathrm{mSv}$ average annual background radiation in the $\mathrm{UK}, 0.02 \mathrm{mSv}$ for a chest $\mathrm{X}$-ray, up to 8 $\mathrm{mSv}$ for a CT scan of the chest. Because the half-life of $18 \mathrm{~F}$ is about two hours, the prepared doses decay significantly during the working day. If the FDG is delivered to the scanning suite in the morning, the specific activity falls during the day, and a relatively larger volume of radio pharmaceutical must be injected in later patients to deliver the same radioactive dose $\mathrm{e}^{4}$.

\section{Bangladesh Perspective:}

Bangladesh, having a population of 160 million people should have several PET Scanners. Our neighboring countries like India, Pakistan, Thailand, Singapore etc. have PET Scanners running for last several years. Dhaka, being central and capital city of Bangladesh, harboring about 15 million people needs to have regular PET scanner services to ensure modern treatment in certain fields. The modern PET system consists of a cyclotron machine, PET scanner, accompanied by CT or MRI setting, usually CT scanner. The cyclotron machine produces radionuclide and is the costlier part of the system. A cyclotron machine can produce the amount of radionuclide which can be supplied to 4-6 PET-CT scanners. So, by a single cyclotron, several PET-CT scanners can run. Once the cyclotron is installed, a few more PET-CT may be installed. First PET/CT Scanner in Bangladesh has been introduced by a private multidisciplinary tertiary level hospital United Hospital, Gulshan, Dhaka in the year 2011. United Hospital has set up the first cyclotron in its campus in Bangladesh. The cyclotron produces shortlived radioisotopes needed for medical imaging using positron emission tomography (PET). The staff of cyclotron facility includes experienced radio chemist and radio pharmacist capable of developing novel PET imaging tracers to expand the scope of imaging at United Hospital and surrounding medical institutions ${ }^{19}$. The Traffic jam in Dhaka city should be considered vigorously for transportation of radionuclide from cyclotron production site to the PET-CT installation centers because the half life of 18-FDG is less than 2 hours. The radionuclide (18-FDG) produced at cyclotron production site can be transported to the adjacent private and government medical institutions if they install PET/CT Scanner. The cost of PET imaging is still high (approximately Taka 65000 per scanning procedure) and should be subsidized by government.

\section{Conclusion:}

The history and evaluation of PET imaging shows continuous improvement in sensitivity and resolution. At present, PET scanning is mainly used in diagnosis, staging and monitoring of treatment of cancer as well as detection of cancer recurrence. The PET imaging system usually includes also simultaneous CT scanner in the same gantry. The PET scan demonstrates the biological function of the body tissues, while the CT scan provides information about the body's anatomy such as size, shape and location of the lesion. By combining these two technologies, physicians can more accurately diagnose and monitor diseases such as cancer, heart diseases, and certain brain disorders. The wide spread use of CT, MRI and their versions - all are concerned with analysis of structural lesions; none carrying any significant physiological data. Understanding of molecular biology and metabolism in disease states, development of suitable radiotracers, combinations of PET with other imaging modalities like CT and MRI can improve more in the diagnosis and treatment of certain diseases.

\section{References:}

1. Alam HM, Jean CU, Leon SM, Nicholas JB.Radionuclide imaging: general principles. In: Grainger RG, Allison D (Editors). Diagnostic Radiology, $4^{\text {th }}$ Edition; London: Churchill Livingstone; 2003: 137- 140.

2. Positron (Encyclopedia article). Microsoft (R) Encarta (R) Reference Library 2005 (C) 1993-2004 Microsoft Corporation: Encarta Reference Library Premium 2005 DVD.

3. Selman J, Thomas CC. The fundamental of X-ray and radium physics, $7^{\text {th }}$ Edition; Springfield: Charles $C$ Thomas publisher; 1985: 491-535. 
4. Positron emission tomography (http://en. wikipedia.org/wiki/ positron_emission_tomography (PET) imaging; Accessed on 24 October 2006).

5. Radiological Society of North America. Radiology info positron emission tomography (PET imaging); Radiological Society of North America, Inc. (RSNA); (Reviewed on 21 April 2005).

6. Curry TS, Dowey JE, Murr RC. Christensen's Physics of Diagnostic Radiology, $4^{\text {th }}$ Edition; Philadelphia: Lea \& Febiger; 1990: 242-49.

7. Brownell GL. A history of positron imaging. Physics Research Laboratory, Massachusetts General Hospital, Division of Radiological Sciences, Massachusetts Institute of Technology 1999-10-15. (http: //www.mit.edu/ glb/alb.html; Accessed on 11 October 2007).

8. Jones AP, Jeukins JPR, Robinson PJA, et al. Recent technical advances. In: Sutton D (Editor). Textbook of Radiology and Imaging, $7^{\text {th }}$ Edition; London: Churchill Livingstone; 2003: 1829-31.

9. Introduction to PET physics: Positron emission tomography (PET) in medical imaging. (file://H: $\backslash U W$ Introduction to PET Physics - files\section 1.htm; Accessed on 17 August 2006).

10. Burtis CA, Ashwood ER. Tiez fundamentals of clinical chemistry, $5^{\text {th }}$ Edition; Philadelphia: WB Saunders Company; 2001: 574 .

11. Brownell GL. First positron imaging device. Physics Research Laboratory, Massachusetts General Hospital, Division of Radiological Sciences, Massachusetts Institute of Technology 1999-10-15. (http: //www.mit.edu/ glb/alb.html; Accessed on 11 October 2007).
12. Brownell GL. First multiple detector positron imaging device1962. Physics Research Laboratory, Massachusetts General Hospital, Division of Radiological Sciences, Massachusetts Institute of Technology 1999-10-15. (http://www.mit. edu/ glb/alb.html; Accessed on 11 October 2007).

13. Brownell GL. PC-1 The first tomographic imaging device and the first computed tomographic imaging device (PET 1968-1971), Physics Research Laboratory, Massachusetts General Hospital, Division of Radiological Sciences, Massachusetts Institute of Technology 1999-10-15. (http: // www.mit.edu/ glb/alb.html; Accessed on 11 October 2007).

14. Underwood SR, Anagnostopoulos C. Nuclear cardiology. In: Grainger RG, Allison D (Editors). Diagnostic Radiology, $4^{\text {th }}$ Edition; London: Churchill Livingstone; 2001: 721-37.

15. Nelson DL, Cox MM. Lehlinger's Principle of Biochemistry, $4^{\text {th }}$ Edition; New York: WH Freeman \& Company; 2005: 533.

16. Innes JA, Reid PT. Respiratory disease. In: Boon NA, Colledge NR, Walker BR, Hunter JAA (Editors). Davidson's Principles and Practice of Medicines, 20 ${ }^{\text {th }}$ Edition; London: Churchill Livingstone; 2006: 654.

17. Sawada SG. Positron emission tomography for assessment of viability. Curr Opin Cardiol 2006 Sept; 21(5): 464-68.

18. Nishimura RA, Gibbons RJ, Glockner JF, Tajik AJ. Noninvasive cardiac imaging: echocardiography, nuclear cardiology, and MRI/CT imaging. In: Kasper DL, Fauci AS, Longo DL (Editors). Harrison's Principles of Internal Medicine, $16^{\text {th }}$ Edition; New York: McGraw Hill Companies; 2005: 1320-27.

19. First PET/CT in Bangladesh: Nuclear Medicine; United Hospital Ltd. (http//www.unlbd.com/services. Accessed on 1 July 2011). 